\title{
Patterns of sensory loss following fractional posterior fossa $V$ th nerve section for trigeminal neuralgia
}

\author{
M HUSSEIN, * LA WILSON, $†$ R ILLINGWORTH \\ From the Regional Departments of Neurology and Neurosurgery, Central Middlesex Hospital, London, UK
}

SUMMARY Twenty-five patients with trigeminal neuralgia have been treated by fractional posterior fossa $V$ th nerve section. The procedure is shown to be effective in relieving pain, preserving corneal sensation and the motor root. Nineteen patients showed little loss of light touch sensation, and in 15 of these there was dissociation of sensory loss to pinprick and light touch. The results support the concept that there is some anatomical separation of fibres subserving light touch and pain in the posterior root of the nerve.

\begin{abstract}
Although carbamazepine (Tegretol) is effective for most patients with trigeminal neuralgia, surgery is still required where the response is poor or side effects occur. In these circumstances percutaneous electrofrequency thermocoagulation of the nerve ${ }^{1}$ is superseding the long-established methods of middle fossa extradural nerve section and chemical injection of the ganglion. ${ }^{2}$ An alternative is the posterior fossa operation first proposed by Dandy. ${ }^{3}$ This is the only surgical method of curing trigeminal neuralgia without producing sensory loss provided vascular cross-compression can be found and relieved. ${ }^{4}$ The original advantages claimed for the posterior fossa approach were that the arrangement of the nerve fibres allowed some preservation of light touch and that keratitis did not occur when subtotal section of the nerve was performed. ${ }^{5}$ It has been suggested that some light touch sensation is carried by a group of fibres called accessory, ${ }^{5}$ or intermediate, ${ }^{6}$ lying medially between the main sensory root and the motor root. However, the sensory function of these fibres has been denied, ${ }^{78}$ and the ability of posterior fossa Vth nerve section to produce dissociated sensory loss is not well documented. ${ }^{79}$ This paper describes the results, including the patterns of sen-
\end{abstract}

Present Address: *Department of Surgery, Faculty of Medicine, University of Khartoum, P.O. Box 102, Khartoum, Sudan, and tDepartment of Neurology, Royal Free Hospital, Pond Street, London NW3.

Address for reprint requests: Mr R Illingworth, Central Middlesex Hospital, Acton Lane, London NW10 7NS, UK.

Received 1 February 1982

Accepted 5 May 1982 sory loss, produced in 25 consecutive patients who had fractional posterior fossa Vth nerve section performed for trigeminal neuralgia.

\section{Materials and methods}

Twenty-five patients with apparently idiopathic trigeminal neuralgia have been treated by fractional posterior fossa Vth nerve section between June 1972 and July 1978. Eleven patients were male and 14 female. The ages ranged from 35 to 76 years (mean 59 years). Fifteen patients were over the age of 60 , and three over the age of 70 years.

Seventeen patients had trigeminal neuralgia affecting the second and third divisions, two in the second division alone and two in the third division alone. In two patients the first and second divisions were affected, in one all divisions, and in one only the ophthalmic division. Preoperative sensation was normal in 21 patients. Four patients with mild sensory abnormality had had previous peripheral sections or ganglion injections. Two of these had slight depression of the corneal reflex. One patient had a mild right facial paresis and was later found to have an ectatic basilar artery looping laterally into the cerebellopontine angle.

The aim was always to relieve trigeminal neuralgia by dividing sufficient of the nerve to produce analgesia in the part of the face where the pain was experienced, but sparing the accessory fibres in the hope of preserving some light touch sensation. The patients were placed in the lateral decubitus position for operation. Pre-operative steroid medication and hyperventilation anaesthesia were used to allow the Vth nerve to be exposed through a small retromastoid craniectomy with minimal cerebellar retraction. Magnification at $\times 10$ or $\times 16$ was used to identify the components of the Vth nerve and any other local structures. The nerves were divided flush with the side of the pons with scissors or sharp hook starting laterally. In one patient (No 12) with ophthalmic pain, the section was made from 
the medial side. All the operations were performed by one surgeon (RI), and each operation record included an estimate of the proportion of the main sensory root sectioned. Detailed post-operative sensory testing was performed in all patients before discharge from hospital and again at follow up attendance. Seventeen patients were reexamined, three to nine years after operation by a neurologist (LAW), who was unaware of the extent of section or the previous sensory findings. Three patients (Nos 4,12 , and 24 ) were not re-examined because they had died of causes unrelated to trigeminal neuralgia or its treatment 15, 54 and 99 months after operation. They had been followed up to the time of death. Five patients were not reexamined because they were unable, or in one case unwilling to attend (Nos 8, 13, 14, 20, 22). The patients were tested for sensation to pinprick, temperature, cotton wool and two-point discrimination. The temperature loss largely corresponded with loss to pinprick. Two-point discrimination was used to confirm the sensory findings to light touch: Corneal reflex and jaw muscles were also examined.

The presence of pain and other subjective sensory dis- turbance has been determined by letter follow-up and in most by outpatient attendance. Pain or subjective sensory symptoms have been classified as mild if they do no cause inconvenience, interfere with patients' lives or require any medication. The symptoms have been called troublesome if they interfere with patients' lives, require medication or have led to outpatient attendance requesting further treatment. All the surviving patients have been followed up to the time of writing for at least three years since operation (38-109 months, mean 79 months).

\section{Results}

\section{SENSORY LOSS}

The sensory loss to pain and light touch in each patient is shown in fig 1 . The faces are show as if facing towards the reader and the number of each patient is given below each pair of half faces. It is seen that increasing section of the posterior root from laterally produced increasing analgesia, usually

\section{Estimated}

\section{section \%}
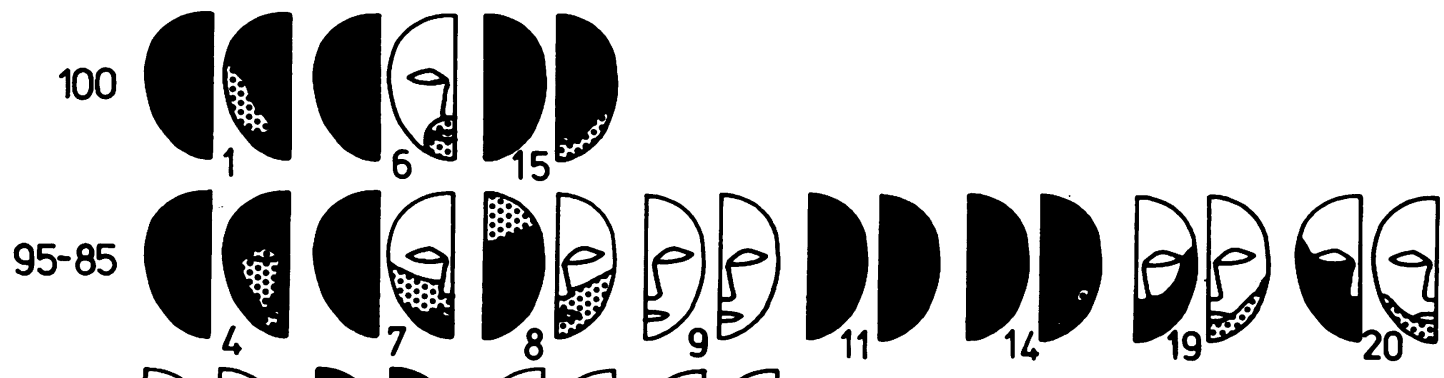

75
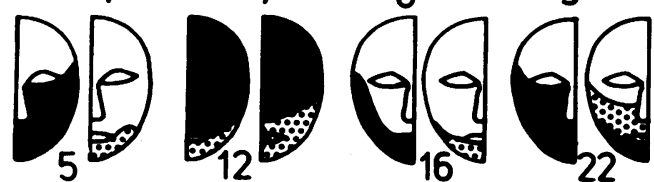

66
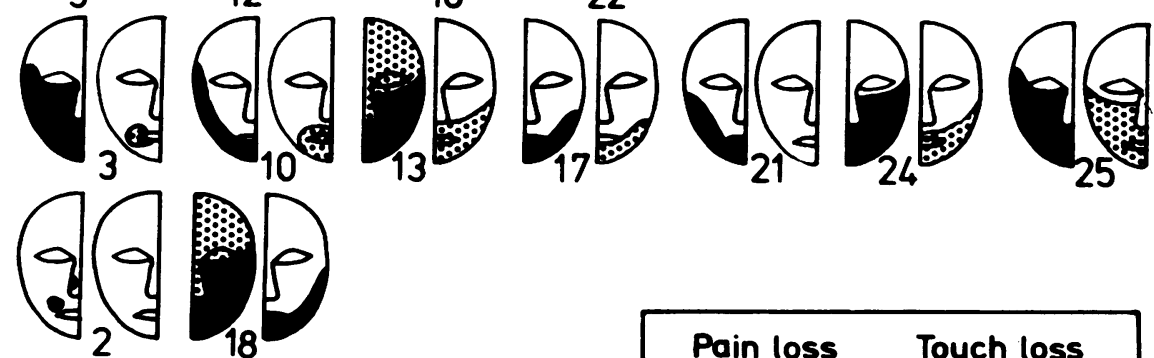

33
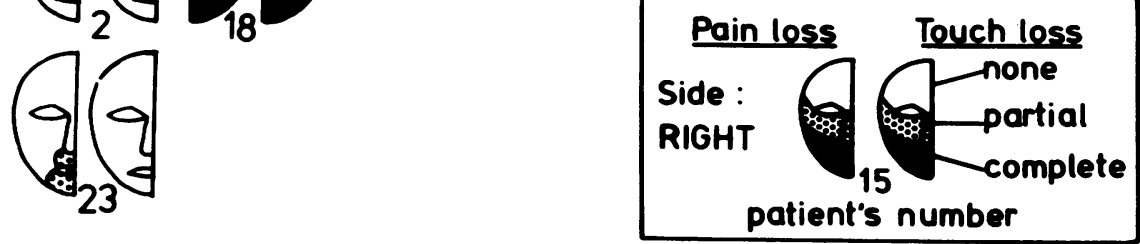

Fig 1 Sensory loss to pain and to light touch produced in 25 patients after fractional posterior fossa Vth nerve section. 
with a well-defined upper margin ascending on the face from mandibular to ophthalmic divisions. Often the amount of analgesia produced was less than might be expected from the estimate of the extent of nerve sectioned. Fifteen patients showed marked differences between the extent of loss to pain and touch. This dissociation of sensory loss was found where the extent of analgesia was small, involving only the mandibular divisions (Nos 10, 16, 19, 21), and also in patients with mandibular and maxillary analgesia (Nos 3, 5, 8, 13, 18, 20, 22, 24, 25). Marked dissociation of sensory loss with good preservation of light touch sensation also was found in two patients with complete or marked hemifacial analgesia (Nos 6,7), but six other patients with complete hemifacial analgesia also had complete or virtually complete hemifacial anaesthesia (Nos 1, 4, $11,12,14,15)$. The pattern. of sensory loss in patient No 12 was unlike that of any other and was produced by a section of the medial $75 \%$ of the major portion of the nerve in a patient with ophthalmic division pain.

In three patients recent re-testing showed either no sensory loss to either pain or touch (No 9), or only minor localised sensory loss to pain (Nos 2, 23). One of these (No 23) had had the smallest section estimated at $33 \%$ and this had been found at follow up to have produced mandibular anaesthesia, which at re-examination had shrunk to some peri-oral hypalgesia. Two other patients at initial follow up had extensive analgesia, including the mandibular and most of the maxillary divisions, but with little (No 2) or no light touch loss (No 9). The sensory findings in these two patients when re-examined independently 7 and 8 years later are shown in fig 2 . Five other patients showed lesser reductions in analgesia, in four cases the change being less than one division (Nos 5, 16, 17, 19) and in one case more than one division (No 10), also shown in fig 2 . The initial loss of light touch sensation in these patients usually was not extensive, and this has not changed. No patient has shown any increase in sensory loss.

\section{CORNEAL REFLEX}

In 17 patients the nerve section had no effect on corneal sensation or reflex, which remained unchanged. In eight patients (Nos 1, 4, 7, 10, 11, 12, $14,15)$ post-operative testing showed the corneal reflex to be impaired. One patient (No 10) with intact sensation to both light touch and pain in the ophthalmic division had a reduced corneal reflex. Only one patient with complete pain loss in the ophthalmic division had a preserved corneal reflex (No 6) but the corneal reflex was intact in three patients with partial ophthalmic loss to pain (Nos 8, 13, 18).

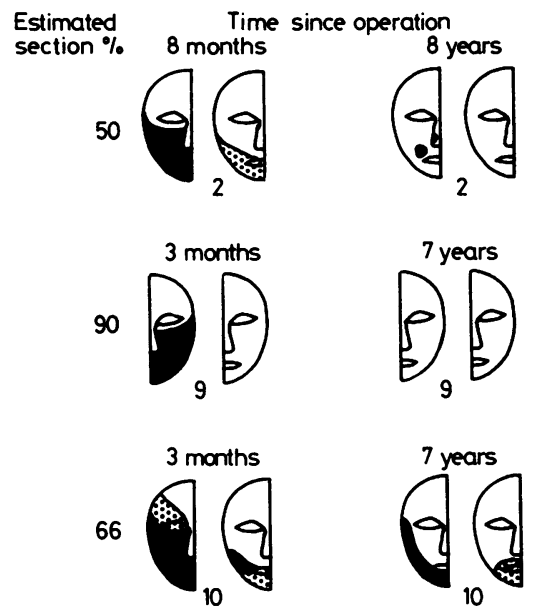

Fig 2 Alterations in sensory loss found in three patients re-examined several months and again several years after fractional posterior fossa Vth nerve section (scheme as in fig 1).

ACCESSORY AND MOTOR FIBRES

Accessory fibres were identified at operation in 21 patients. They were not seen in two and were not mentioned in the operative notes of two. Postoperative motor function of the trigeminal nerve was intact in 24 patients and in one there was no record.

POST-OPERATIVE FACIAL PAIN AND SUBJECTIVE SENSORY SYMPTOMS

No information is available from one patient known to be alive. Of the remaining 24 patients, only two had troublesome persisting sensory symptoms. One of these (No 13) had aching pain and paraesthesia, the other (No 6) paraesthesia only. Five patients (Nos 1, 6, 7, 12, 25) had mild dull pain not requiring any treatment, and six (Nos $1,3,4,5,11,25)$ had trivial paraesthesiae or an awareness of numbness. Pain and other sensory symptoms following operation were commoner in patients who had more extensive sections and had marked sensory loss. The presence or absence of dissociated sensory loss did not appear to be as relevant as the total amount of sensory loss in determining whether the patient had post-operative symptoms.

\section{CLINICAL RESULTS AND COMPLICATIONS}

Trigeminal neuralgia was immediately relieved by operation in all 25 patients. One patient with trigeminal neuralgia involving the second and third divisions with occasional pain in the first division pre-operatively had a recurrence of pain in the first division about a year after section had produced 
analgesia extending up to the eye. His pain has been easily controlled on a small dose of carbamazepine and a further section has not been necessary. Two patients in whom tortuous dilated basilar arteries were elevating the Vth and also the VIIth and VIIIth cranial nerves had transient post-operative facial palsies which recovered after several months, and one also had a transient VIth nerve palsy. One of these patients also had a mild VIIth nerve palsy pre-operatively. About half of the patients developed mild facial herpes, but none involved the eye and no keratitis developed. There was no operative mortality.

\section{Discussion}

The results of selective posterior fossa Vth nerve section described in this small series indicate that the procedure is an effective means of relieving trigeminal neuralgia. Limited section is shown to preserve the motor root and, in many patients, a considerable degree of light touch sensation. This dissociated sensory loss cannot be guaranteed and tends to be less evident when sensory loss to pain involves the ophthalmic division. Corneal reflexes have been preserved in the majority of patients, but this is less likely when the sensory loss to pain involves the ophthalmic division, and particularly when there is also marked loss of light touch sensation. Postoperative facial pain (not trigeminal neuralgia), or paraesthesia, affected two patients. Nine other patients were aware of minor symptoms.

Although these results are acceptable in terms of relief of trigeminal neuralgia and the low incidence of undesirable side effects, they do not in themselves appear to be sufficiently different from other modes of surgical management ${ }^{1011}$ to justify the adoption of posterior fossa $V$ th nerve section on these grounds alone.

Although the more controlled methods of thermocoagulation can result in relative preservation of light touch, there is a tendency for the sensory loss to fade and recurrence of trigeminal neuralgia in $14 \%,{ }^{10} 23 \%{ }^{11}$ and $28 \%$ of patients followed $4 \frac{1}{2}$ to 9 years ${ }^{1}$ has been described. Follow-up exceeding 12 years in one series gave a recurrence rate of $80 \% .{ }^{12}$ Surgical section may have the advantage of producing a more permanent effect, although in this small series three patients showed marked recovery of sensation after 7 or 8 years. With modern anaesthesia and microsurgical techniques the procedure appears safe and operative mortalities of less than $1 \%$ have been described in two series of 200 patients. $^{413}$

The pattern of dissociated sensory loss produced in many patients in this series does suggest that fibres carrying light touch sensation tend to collect in the medial part of the main sensory root as it enters the pons. Whether fibres concerned with light touch occupy the accessory fibres is not entirely clear, although a few patients in whom total or subtotal section of the major root was performed had sufficient preservation of light touch to suggest that this theory may have some basis. ${ }^{14}$ However, if fibres concerned with light touch sensation are concentrated in the medial part of the main sensory root, one might anticipate that section of the medial part of the root would result in more loss of light touch sensation than pain. The result in one patient (No 12) in whom a medial section was performed, does not support that concept. This may be explained by the increased technical difficulty in sectioning the medial part of the nerve, and the likelihood of producing some injury to the remaining lateral part.

The main justification for performing a $V$ th nerve exploration through the posterior fossa must be the hope of producing relief of pain without the necessity of nerve section, through the discovery and successful treatment of vascular cross-compression. ${ }^{4}$ In this series of 25 patients arterial cross-compression was identified in 12 patients in whom there was clear operative evidence that the nerve was indented, displaced or compressed by an arterial loop. Three more patients had arterial loops in contact with the nerve and two patients were found to have the nerve compressed by a local vein. In two the nerve was found to be surrounded by dense arachnoid, and one patient was found to have a small trigeminal neurinoma. In only five patients did the nerve appear to be normal. During the period of this study these findings did not usually alter the pre-operative decision to perform a nerve section, but two later patients in the study had decompressive procedures combined with rather smaller sections than had been planned. These results and increasing experience have led us to prefer vascular decompression as the primary treatment and the results so far justify continuation of that policy. Our experience with selective posterior fossa $V$ th nerve section suggests that this is an acceptable alternative when no treatable anatomical cause is found.

We are grateful to Mrs V Roberts for much secretarial assistance.

\section{References}

'Sweet WH. Treatment of Facial Pain by Percutaneous Differential Thermal Trigeminal Rhizotomy. In: Krayenbuhl H, Maspes PE, Sweet WH, eds. Progress in Neurological Surgery 7. Basel: Karger, 1976:15379. 
${ }^{2}$ Loeser JD. What to do about tic douloureux. JAMA 1978;239:1153-5.

${ }^{3}$ Dandy WE. An operation for cure of tic douloureux: partial section of the sensory nerve at the pons. Arch Surg 1929;18:687-734.

4 Jannetta PJ. Microsurgical approach to the trigeminal nerve for tic douloureux. In: Krayenbuhl $\mathrm{H}$, Maspes PE, Sweet WH, eds. Progress in Neurological Surgery 7. Basel: Karger, 1976:180-200.

5 Dandy WE. The treatment of trigeminal neuralgia by the cerebellar route. Ann Surg 1932;96:787-95.

- Jannetta PJ, Rand RW. Transtentorial retrogasserian rhizotomy in trigeminal neuralgia by microneurosurgical technique. Bull Los Angeles Neurol Soc 1966;31:93-9.

${ }^{7}$ Pelletier VA, Poulos DA, Lende RA. Functional localisation in the trigeminal root. $J$ Neurosurg 1974;40:504-13.

${ }^{8}$ Saunders RL, Sachs E. Relation of the accessory rootlets of the trigeminal nerve to its motor root. A microsurgical autopsy study. J Neurosurg 1970;33:317-24.
9 Provost J, Hardy J. Microchirurgie du trijumeau: anatomie fonctionnelle. Neurochirurgie 1970;16 :459-70.

${ }^{10}$ Nugent GR. Radiofrequency treatment of trigeminal neuralgia. Nursing Times 1978;74:229-30.

"Schürmann E, Schürmann K. Controlled and Partial Percutaneous Electrocoagulation of the Gasserian ganglion in facial pain. In: Penzholz $\mathbf{H}$, Brock $\mathbf{M}$, Hamer J, eds. Advances in Neurosurgery 3. Berlin: Springer-Verlag, 1975:301-13.

12 Menzel J, Piotrowski W, Penzholz H. Long term results of gasserian ganglion electrocoagulation. $J$ Neurosurg 1975;42:140-3.

${ }^{13}$ Hanraets PRMJ. Surgical treatment of trigeminal neuralgia following the method of Dandy. Psychiatr Neurol Neurochir 1970;73:441-6.

${ }^{14}$ Ley A, Montserrat L, Bacci F, Ley A Jr. Clinical and electrophysiological studies on sensory conduction mediated by the accessory rootlets of the human trigeminal nerve. J Neurosurg 1975;42:513-21. 